\title{
Effectiveness of antipsychotics used in first- episode psychosis: a naturalistic cohort study
}

Richard Whale, Michael Harris, Gail Kavanagh, Vijitha Wickramasinghe, Christopher I. Jones, Steven Marwaha, Ketan Jethwa, Nirmalan Ayadurai and Andrew Thompson

\section{Background}

One year of antipsychotic treatment from symptom remission is recommended following a first episode of psychosis (FEP).

\section{Aims}

To investigate the effectiveness of commonly used antipsychotic medications in FEP.

\section{Method}

A retrospective cohort study of naturalistic treatment of patients $(N=460)$ accepted by FEP services across seven UK sites. Treatment initiation to all-cause discontinuation determined from case files.

\section{Results}

Risk of treatment discontinuation is greatest within 3 months of treatment initiation. Risperidone had longest median survival time. No significant differences were observed in time to discontinuation between commonly used antipsychotics on multivariable cox regression analysis. Poor adherence and efficacy failure were the most common reasons for discontinuation.

\section{Conclusions}

Effectiveness differences appear not to be a current reason for antipsychotic choice in FEP. Adherence strategies and weighing up likely adverse effects should be the clinical focus.

\section{Declaration of interest}

R.W., A.T. and S.M. have received research grant, speaker honoraria and conference attendance funding from all companies marketing antipsychotics.

\section{Copyright and usage}

(C) The Royal College of Psychiatrists 2016. This is an open access article distributed under the terms of the Creative Commons Non-Commercial, No Derivatives (CC BY-NC-ND) license.
The efficacy of antipsychotic medication for the treatment of acute psychotic episodes and preventing subsequent relapse has been clearly established in randomised controlled trials. ${ }^{1,2}$ Studies of relative efficacy between medications, when combined by network meta-analysis to enable ranking of effect, demonstrate some differences between medications, such as grouping the efficacy of clozapine, olanzapine, amisulpride and risperidone above other antipsychotics. ${ }^{3}$ Such efficacy studies are most commonly undertaken in chronic stage illness samples however. Studies of antipsychotic efficacy conducted specifically in first-episode psychosis (FEP) and early episode samples generally show fewer differences between existing licenced medications, ${ }^{4-6}$ possibly because efficacy of antipsychotics in treating positive symptoms is overall greater in treatment naïve psychosis. Adverse effects appear more likely in treatment naïve samples however, ${ }^{7}$ which combined with other factors varying between antipsychotic medications ${ }^{3}$ may suggest that differences in treatment effectiveness could be more evident in FEP than efficacy differences. Outcomes of naturalistic effectiveness studies in FEP may importantly inform optimal clinical practice beyond the potentially unrepresentative setting of the randomised efficacy trial. ${ }^{8}$

All-cause time to treatment discontinuation has been accepted as a proxy measure of medication effectiveness, incorporating measure of overall tolerability of the medication, views and judgements of the clinician and patient and clinical efficacy. ${ }^{9,10}$ Pragmatic studies in chronic schizophrenia samples of all-cause time to discontinuation have demonstrated benefit of clozapine over other antipsychotics. ${ }^{9,11}$ Several studies have explored time to treatment discontinuation of prescribed antipsychotics for early episode psychosis. These have included pragmatic randomised prospective studies, ${ }^{10,12-15}$ naturalistic cohort studies ${ }^{16,17}$ and a prescription database study. ${ }^{18}$ Significant differences between discontinuation times of the different medications were observed in at least half of these studies, but consistent rankings of effectiveness across studies were not observed. Most studies do not explore the breadth of oral antipsychotics currently available. Heterogeneity of findings is not clearly explained by study design although olanzapine is ranked at least of equal best continuation rate in each of the randomised prospective studies (despite it having the most associated metabolic adverse effects). This mirrors the later stage illness CATIE study data where olanzapine had the lowest discontinuation rate but the greatest weight gain and dyslipidaemia. $^{19}$

We aimed to further explore the aspects of antipsychotic effectiveness in FEP between commonly used medications in a large, naturalistic clinical sample across different UK FEP services, using all-cause time to discontinuation as a comparator outcome. Our hypothesis, based on these previous studies, was that olanzapine would demonstrate a longer relative time to discontinuation in comparison with other antipsychotic medications.

\section{Method}

\section{Study design}

A large sample retrospective, naturalistic cohort design was adopted to compare the time to all-cause treatment discontinuation of different antipsychotic drugs used in the treatment of an FEP in an antipsychotic-naïve population, over 1 year ( 1 year of treatment being a gold standard in clinical treatment). ${ }^{20}$

\section{Participants}

All patients accepted for treatment by the early intervention for psychosis services at seven sites in the counties of Sussex and Warwickshire and the City of Coventry, England, from 28 February 2009 to 28 February 2012 were recruited for the study. The treatment sites covered the areas of Bognor Regis, Brighton and Hove, Coventry, Eastbourne, Horsham, North Warwickshire, South Warwickshire and Worthing and are a mixture of urban and rural sites including areas with both low and high social deprivation indices. 
Included patients met the criteria of 'FEP' in all seven services during the period of data collection, as determined at clinical interview with illness severity defined as a positive 'CAARMS' (comprehensive assessment of at-risk mental states) threshold for psychosis or a score $>4$ on hallucinations and/or delusions and/or $>5$ on conceptual disorganisation subscales of the PANSS (Positive and Negative Syndrome Scale), and of duration at least 7 days. All patients were aged between 14 and 35 years as per service criteria. Patients were excluded from the study if psychotic symptoms were due to a depressive or primary organic aetiology. Patients were also excluded if they were under the care of the service for less than 1 year, or they were not prescribed antipsychotic medication at all during their treatment with the service. Patients started on longacting injectable medications as their first treatment were also excluded from the sample because of difficulty of comparison with oral medications. Patients were not excluded if other psychotropic drugs (non-antipsychotic mood stabilisers, benzodiazepines, antidepressants, etc.) were used in combination with an antipsychotic Those individuals for whom reliable data concerning type and duration of treatment were not available were also excluded.

Clinicians would have commenced and managed treatment following international and local guidelines for treating FEP patients and using their own clinical experience in collaboration with the views and attitudes of the patient and the patient's family. Similarly, treatment was 'open label' with flexible dosing.

\section{Data procedures}

For each patient, the case notes, prescription cards and electronic records available were examined to determine the first antipsychotic prescribed, the date of treatment onset and the point at which the antipsychotic was discontinued. Raters were all psychiatrists (all authors listed except C.I.J.), and the data entered on a pre-designed form with variables defined.

\section{Outcome measures}

Discontinuation was defined as the first day of a 2-week or longer interruption in treatment adherence as decided by author and academic peer consensus. Dates of treatment discontinuation were ascertained by careful scrutiny of recorded clinical notes. Where only approximations were available (e.g. treatment stopped 'a few weeks ago'), prescribing clinicians were contacted to improve precision. Standard dates were used when periods of the month were referred to. Where greater clarity could not be sought, estimates were made by agreement reached by a panel of three of the researchers, or data were entered as 'unavailable' if this was not possible. Where a cross-titration of medication was initiated the date of discontinuation was taken as the first day of the crosstitration. If patients were lost to follow-up (e.g. disengaged from treatment before completing a year of medication), they were excluded from the cohort but included in an intention-to-treat analysis.

All raters undertook an interrater reliability exercise. Records of 10 patients were randomly chosen and a chronology of the prescribing transcribed by the clinician who had initially rated the case. The accuracy of this rating, as judged by the time to discontinuation in days, was assessed based on these transcriptions by all other raters.

The causes for treatment discontinuation were recorded for analysis and comparison between antipsychotic cohorts. These were divided into 'clinician managed' - where the clinician was informed and the discontinuation was planned by the clinician and 'patient managed' - where the clinician was not informed. Reasons for discontinuation were then categorised by the primary reason for patient or clinician dissatisfaction with the medication (such as lack of efficacy, adverse effects or poor adherence without clear association with medication factors). Where adverse effects were the main factor, these were also classified as sedation, sexual dysfunction, extrapyramidal dysfunction, weight gain, agitation or 'other'. ${ }^{21}$ In cases where more than one reason was given for treatment attrition, two researchers determined a consensus rating of the most important reason based on the clinical file, requesting input from the prescribing clinician if possible or a third clinician if necessary to reach majority consensus.

\section{Statistical methods}

Data from each group were analysed following intention-to-treat principles. The median survival time was calculated for specified medications, referring to the number of days when $50 \%$ of the cohort had discontinued that treatment. The advantage of this measurement is that it is not dependent on length of follow-up time (some patients continuing treatment beyond 12 months) and can be compared with median survival times from other studies with different follow-up periods. 'Mean time to patient discontinuation' is often used in studies but is dependent on discontinuation rate, which is relative to the follow-up period used. Cox proportional hazards regression analysis was conducted to determine the impact on time to discontinuation of antipsychotic type, age, gender, duration of untreated psychosis (DUP; defined as the time from date of psychotic symptom onset to the date of medication commencement), clinical role of first prescriber and region of initiation on the survival function. Data were analysed using Stata version 14.1.

\section{Ethics}

Locality governance approval was attained for this study on the basis of service evaluation examining how standard care is delivered where decisions regarding the prescribing and administering of treatment were made jointly by the clinician and patient.

\section{Results}

\section{Study sample and baseline characteristics}

Five hundred and ten patients met the inclusion criteria of FEP for treatment by the early intervention services in this study, within the designated time period. Fifty patients were excluded from this study as per designed method, owing to not starting an oral antipsychotic medication or relevant baseline data were lacking (Fig. 1). Olanzapine, quetiapine, risperidone and aripiprazole were the first-line treatments chosen for $97.8 \%$ of patients, comprising $40.2,25.2,19.1$ and $13.3 \%$ of the sample, respectively. For 28 patients, follow-up data were not available due to disengagement with services or leaving the locality (before 1 year of treatment or until treatment was discontinued), resulting in a final analysis sample of 427. No deaths of any cause were observed in available data. The proportion of patients without follow-up data was similar for the four antipsychotics, ranging from $3 \%$ for olanzapine to $9 \%$ for risperidone.

Baseline characteristics of the study sample are shown in Table 1. The greatest proportion of the sample was male and had antipsychotic medication initiated in a community $v$. an acute care setting. Only $30 \%$ had medication initiated by early intervention services. Median age, median DUP and role of prescriber were all significantly different between the four most likely prescribed antipsychotic medications (all $P<0.001$ ). Those prescribed olanzapine had a notably lower median DUP and were most likely to be managed by acute care services (in-patient or community crisis team) than other groups.

Comparison between baseline variables of included and excluded cases revealed no significant difference between age, 


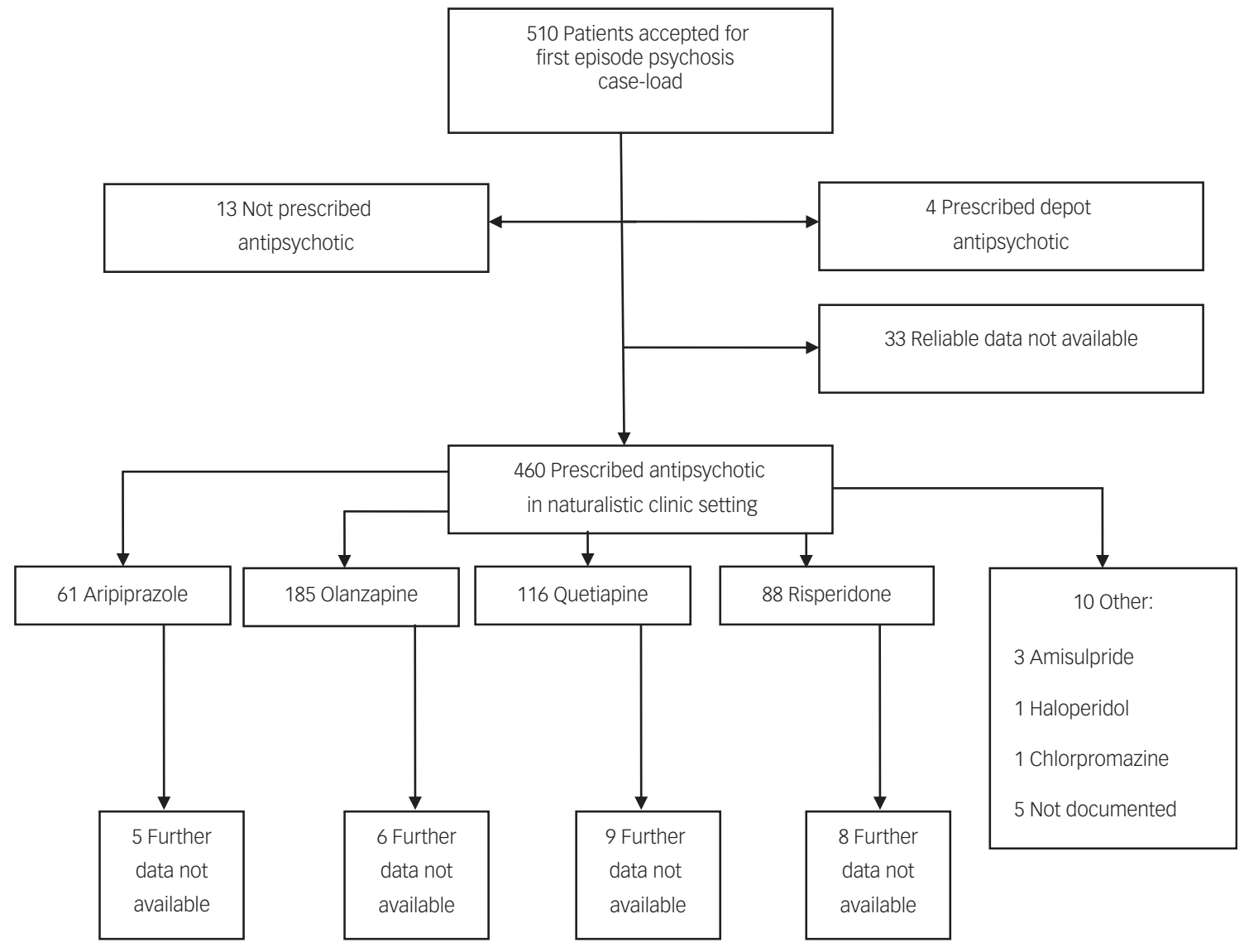

Fig.1 Study recruitment flow chart.

DUP, gender and prescriber role. A significant difference was revealed between geographical areas, with more missing data in Warwickshire than Sussex.

\section{Interrater reliability}

For the interrater reliability exercise, based on time to discontinuation in days between the raters, the intra-class correlation coefficient was very high (0.99) suggesting high reliability of ratings.

\section{Time to discontinuation}

In the whole cohort, $73.3 \%$ of patients discontinued their first prescribed antipsychotic within 12 months. The all-cause rate of discontinuation within 12 months from initiation of medication and median survival times by antipsychotic medication are shown in Table 2. Although the ranking of lowest discontinuation rates and longest time to discontinuation was risperidone, quetiapine, aripiprazole then olanzapine, both favouring risperidone, no statistically significant differences were found between medications. The survival distribution curves of the four medications are plotted in Fig. 2.

Multivariable Cox regression analysis revealed no significant association between medication type and time to discontinuation, using olanzapine as the reference category as per our hypothesis (Table 3). A significant effect of both DUP years (HR 0.87, 95\% CI 0.77 to $0.98, P=0.028$ ) and region of first prescription (Sussex $v$. Warwickshire; HR $1.50,95 \%$ CI 1.05 to $2.15, P=0.026$ ) on survival was demonstrated. The discontinuation rate was reduced by $0.0027 \%$ per week (or $13 \%$ per year) of DUP. Discontinuation rate was $50 \%$ higher in Sussex $v$. Warwickshire. No significant effect of age, gender or prescriber role was observed.
Overall, $60.2 \%(189 / 314)$ of patients discontinued treatment aided by a clinician $v$. those stopping medication themselves (Table 2). No significant difference was observed for this variable between the four most commonly prescribed antipsychotic medications (for Chi-squared test, $P=0.13$ ).

The primary reasons for discontinuation of each antipsychotic group recorded by clinicians are shown in Fig. 3. Of all discontinuations (excluding 66 missing values), 34.4\% were attributed to poor patient adherence (without clear attribution to medication efficacy or adverse events), and this was the primary reason given for patients discontinuing for all antipsychotic groups. The next commonest whole group reason was poor efficacy $(20.5 \%)$.

After poor adherence, the magnitude of reasons for discontinuation by drug (over $5 \%$ of the individual drug sample) was ranked: for aripiprazole, poor efficacy joint with agitation/restlessness, then extrapyramidal side-effects; for olanzapine, weight gain joint with poor adherence, sedation and then poor efficacy; for quetiapine, sedation, poor efficacy and then weight gain; and for risperidone, poor efficacy, raised prolactin, sedation and then weight gain. Relative proportions of these causes by drug are shown in Fig. 3. Of note, olanzapine and risperidone had the lowest rates of poor adherence.

\section{Discussion}

In a large naturalistic FEP cohort, we demonstrate a high rate of all-cause discontinuation (whether switching to another 


\begin{tabular}{|c|c|c|c|c|c|c|c|c|c|}
\hline & & Overall & Aripiprazole & Olanzapine & Quetiapine & Risperidone & Other $^{a}$ & Statistical test & $P$ \\
\hline \multirow{3}{*}{$\begin{array}{l}\text { Age entering EIP } \\
\text { case-load (years) }\end{array}$} & Median & 22.0 & 21.0 & 23.0 & 22.0 & 21.0 & 24.0 & \multirow[t]{3}{*}{ Kruskal-Wallis } & \multirow[t]{3}{*}{0.000} \\
\hline & IQR & 19.0-27.0 & $19.0-26.0$ & $20.0-28.0$ & $19-26.5$ & $18.0-24.0$ & $21.0-25.0$ & & \\
\hline & $n$ & 421 & 55 & 178 & 104 & 79 & 5 & & \\
\hline \multirow{3}{*}{$\begin{array}{c}\text { Duration of untreated } \\
\text { psychosis (weeks) }\end{array}$} & Median & 5.0 & 8.6 & 3.0 & 6.1 & 8.6 & 26.0 & \multirow[t]{3}{*}{ Kruskal-Wallis } & \multirow[t]{3}{*}{0.000} \\
\hline & $\mathrm{IQR}$ & $1.0-22.3$ & 2.7-23.1 & $1.0-11.7$ & $1.3-30.0$ & $2.0-48.6$ & $7.9-26.9$ & & \\
\hline & $n$ & 384 & 45 & 165 & 97 & 72 & 5 & & \\
\hline \multirow[t]{3}{*}{ Gender } & $\%$ male & 65.1 & 55.4 & 67.6 & 62.6 & 68.8 & 80.0 & \multirow[t]{3}{*}{ Chi-squared } & \multirow[t]{3}{*}{0.311} \\
\hline & $n$ male & 278 & 31 & 121 & 67 & 55 & 4 & & \\
\hline & $n$ overall & 427 & 56 & 179 & 107 & 80 & 5 & & \\
\hline \multirow{5}{*}{$\begin{array}{c}\text { Clinical role of } \\
\text { prescriber }\end{array}$} & Acute services\% & 43.0 & 37.5 & 59.0 & 28.3 & 31.3 & 33.3 & & \\
\hline & Community (non-EIP)\% & 24.4 & 28.6 & 20.8 & 28.3 & 22.5 & 66.7 & & \\
\hline & EIP\% & 30.3 & 32.1 & 17.4 & 41.5 & 43.8 & 0.0 & \multirow[t]{3}{*}{ Chi-squared } & \multirow[t]{3}{*}{$<0.001$} \\
\hline & $\mathrm{GP}^{\mathrm{a}}$ & 2.4 & 1.8 & 2.8 & 1.9 & 2.5 & 0.0 & & \\
\hline & $n$ overall & 423 & 56 & 178 & 106 & 80 & 3 & & \\
\hline \multirow[t]{4}{*}{ Region } & \% Sussex & 78.5 & 76.8 & 82.7 & 80.4 & 72.5 & 0.0 & & \\
\hline & $n$ Sussex & 335 & 43 & 148 & 86 & 58 & 0 & & \\
\hline & $\%$ Warwickshire & 21.6 & 23.2 & 17.3 & 19.6 & 27.5 & 100.0 & \multirow[t]{2}{*}{ Chi-squared } & \multirow[t]{2}{*}{0.284} \\
\hline & $n$ Warwickshire & 92 & 13 & 31 & 21 & 22 & 5 & & \\
\hline
\end{tabular}

medication or not) of the initially prescribed antipsychotic of $73.3 \%$ by 1 year. Four antipsychotics (aripiprazole, olanzapine, quetiapine and risperidone) accounted for $97.8 \%$ of prescriptions for first-line treatment of FEP. Risk of discontinuation was highest in the first 3 months of treatment for all medications. Although risperidone had a longer median time to discontinuation than other medications, multivariable Cox regression analysis revealed no significant effect of medication type on all-cause time to discontinuation. Significant differences were observed between prescribed medication groups for age, DUP and role of initiating prescriber. Only DUP and geographical site had significant influence on discontinuation. The most prevalent reason for discontinuation was 'poor patient adherence', grouped as independent of clearly associated adverse effects.

DUP has a consistently demonstrated effect on FEP outcome, and a longer DUP may be explained by negative symptoms. ${ }^{22}$ Negative symptoms have an arguably poor response to currently licensed antipsychotic medications, ${ }^{23}$ which may account for earlier discontinuation in those with a longer DUP. Although olanzapine did not have a significantly different discontinuation rate from other medications, it appeared to be most used in acute services, possibly a marker for a more severely unwell group. This could explain olanzapine's lower ranked median discontinuation time, although prescriber role did not have significant effect in the multivariable analysis. The olanzapine-exposed group also had a shorter DUP than other medications but this did not positively bias its comparative discontinuation rate. The significant effect of region on discontinuation is unexpected and reasons are unclear from this data set. The other difference observed between regions was a larger missing data rate in Warwickshire, which does not have obvious association with discontinuation time. Variables not explored, such as ethnicity, may influence this effect of region.

\section{Comparison with previous studies}

In comparison with other studies that have described all-cause medication discontinuation rates in FEP populations, our overall rate was higher than reported in randomised studies (all-cause discontinuation at 12 months for Kahn et al ${ }^{10}$ was $41.6 \%$, McEvoy et $a l^{12} 70.3 \%$, Crespo-Facorro et al $^{14} 58.6 \%$ and San et $a l^{15} 64 \%$ ). The naturalistic follow-up study by Tiihonen et al described a rapid early attrition with discontinuation rate of $54.3 \%$ at 30 days (a much greater rate than we observed, although their sample was post in-patient admission), and $92.5 \%$ at a mean follow-up of 2 years (direct comparison with our data not possible). ${ }^{16}$ Also naturalistic, Liew et al described a $90 \%$ all-cause discontinuation rate by 18 months, roughly $80 \%$ by 12 months (extrapolated from published report). ${ }^{17}$ This implies that the boundaries of and nature of patients recruited to a randomised study enhance antipsychotic adherence in comparison with a naturalistic study. It is also arguable that our sample, using FEP criteria rather than

\begin{tabular}{|c|c|c|c|c|c|c|c|c|c|}
\hline & & Overall & Aripiprazole & Olanzapine & Quetiapine & Risperidone & Other $^{\mathrm{a}}$ & Statistical test & $P$ \\
\hline \multirow{3}{*}{$\begin{array}{l}\text { Discontinuation within } \\
12 \text { months }\end{array}$} & $\%$ & 73.3 & 76.8 & 78.2 & 69.2 & 66.3 & 60.0 & \multirow{3}{*}{ Chi-squared } & \multirow{3}{*}{0.136} \\
\hline & $n$ & 313 & 43 & 140 & 74 & 53 & 3 & & \\
\hline & $n$ overall & 427 & 56 & 179 & 107 & 80 & 5 & & \\
\hline \multirow{3}{*}{$\begin{array}{l}\text { Time to discontinuation } \\
\text { (days) }\end{array}$} & Median & 75.0 & 73.0 & 70.0 & 77.0 & 87.0 & 28.0 & \multirow{3}{*}{ Kruskal-Wallis } & \multirow{3}{*}{0.997} \\
\hline & IQR & 36.0-154.0 & $35.0-176.0$ & $41.5-145.5$ & $30.0-145.0$ & $30.0-165.0$ & 10.0-37.0 & & \\
\hline & $n$ overall & 427 & 56 & 179 & 107 & 80 & 5 & & \\
\hline \multirow{3}{*}{$\begin{array}{l}\text { Clinician managed } \\
\text { discontinuation? }\end{array}$} & $\%$ yes & 60.2 & 50.0 & 63.1 & 52.8 & 68.5 & 100.0 & \multirow{3}{*}{ Chi-squared } & \multirow{3}{*}{0.133} \\
\hline & $n$ yes & 189 & 22 & 89 & 38 & 37 & 3 & & \\
\hline & $n$ overall & 314 & 44 & 141 & 72 & 54 & 3 & & \\
\hline
\end{tabular}




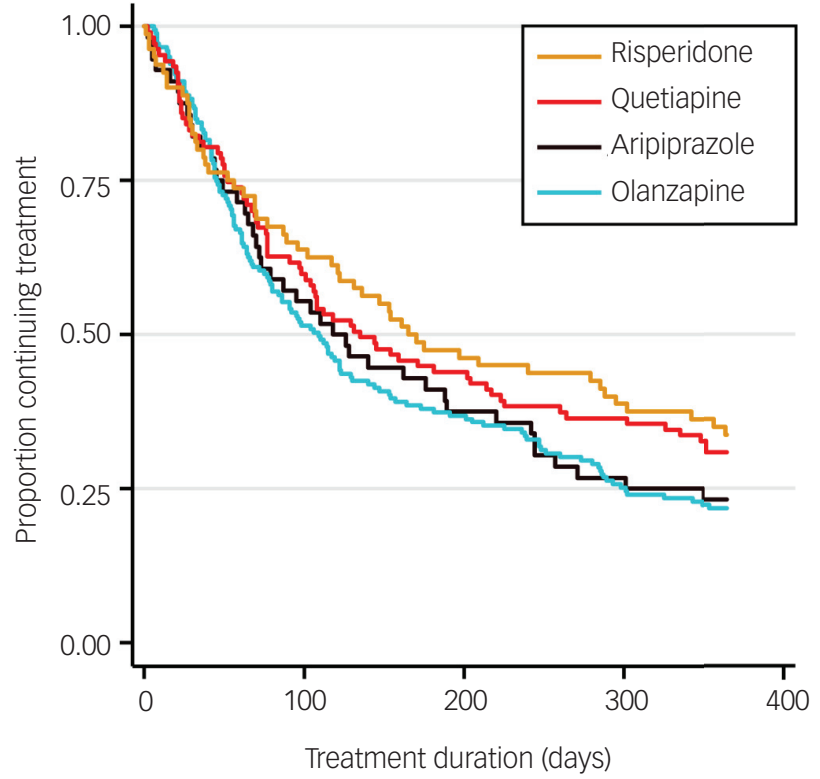

Fig. 2 Kaplan-Meier survival estimates for time to all-cause treatment discontinuation by antipsychotic medication.

specific ICD-10 or DSM-IV diagnoses, was less stringent for inclusion, and possibly overall less severely unwell, with more likelihood of remaining well after a single psychotic episode and therefore more likely to discontinue medication.

Despite differences in ranking of order of rate of discontinuation (and median discontinuation time), our finding of no significant difference between commonly used medications on the survival function in multivariable analysis is consistent with previous studies. Kahn et al conducted a randomised, unblinded, pragmatic study in an analysis sample of 498 patients. ${ }^{10}$ They found relatively lower rates of all-cause discontinuation at 12 months for olanzapine (33\%) than quetiapine (53\%), amisulpride $(40 \%)$ and ziprasidone (45\%) and a notably higher discontinuation rate with haloperidol (72\%). Survival analysis showed significant differences when comparing all medications but no significant difference was observed between olanzapine and quetiapine. ${ }^{20}$ McEvoy et al randomised treatment to olanzapine, quetiapine and risperidone in a patient sample with FEP, finding no significant difference in all-cause discontinuation at 52 weeks

\begin{tabular}{|c|c|c|c|}
\hline & Hazard ratio & $95 \% \mathrm{Cl}$ & $P$ \\
\hline \multicolumn{4}{|c|}{ Medication (olanzapine reference category) } \\
\hline Aripiprazole & 1.06 & $0.72-1.57$ & 0.756 \\
\hline Quetiapine & 0.94 & $0.69-1.28$ & 0.677 \\
\hline Risperidone & 0.84 & $0.59-1.20$ & 0.348 \\
\hline Age & 1.02 & $0.99-1.04$ & 0.156 \\
\hline DUP years & 0.87 & $0.77-0.98$ & 0.028 \\
\hline \multicolumn{4}{|l|}{ Gender } \\
\hline Male & 1.06 & $0.82-1.36$ & 0.652 \\
\hline \multicolumn{4}{|c|}{ Prescriber role (acute services reference category) } \\
\hline Community (non-EIP) & 0.95 & $0.70-1.23$ & 0.722 \\
\hline EIP & 0.86 & $0.63-1.18$ & 0.346 \\
\hline GP & 1.22 & $0.56-2.64$ & 0.613 \\
\hline \multicolumn{4}{|l|}{ Region } \\
\hline Sussex & 1.50 & $1.05-2.15$ & 0.026 \\
\hline
\end{tabular}

but a numerically lower rate with olanzapine (68.4, 70.9 and $71.4 \%$, respectively). ${ }^{12}$ Crespo-Facorro et al followed up a sample of 174 patients with first-episode non-affective schizophrenia spectrum disorders, randomised to haloperidol, risperidone and olanzapine, and although they found discontinuation rates at 1 year were significantly higher for haloperidol, the rates for risperidone and olanzapine were not significantly different. ${ }^{14}$ This lack of difference was sustained by 3 years' follow-up. ${ }^{24}$ Johnsen et al randomised 213 successive admissions with a wide range of diagnosed psychotic disorders (including depressive and drug-induced psychosis) at an emergency ward in Norway to four commonly used antipsychotics. ${ }^{13}$ Included participants were not clearly FEP however, with only $44 \%$ of participants being antipsychotic naïve. At 1-year follow-up, study dropout rates were high, but by an intention-to-treat model, ranking of greatest likelihood of continuation was quetiapine, olanzapine, ziprasidone then risperidone, but not of different enough magnitude to render significance.

In contrast with our results, Tiihonen et al followed up a naturalistic cohort of first hospitalised patients for schizophrenia and schizoaffective disorder (interpreted as a first episode of schizophrenia) in Finland and found olanzapine was significantly less likely to be discontinued within 30 days of discharge $v$. risperidone and less associated with rehospitalisation than risperidone over a mean 2 years follow-up. ${ }^{16}$ In comparison, discontinuation within 30 days was very similar for all medications in our sample. Twelve-month discontinuation comparison was not reported by Tiihonen et al and neither quetiapine nor aripiprazole were commonly used. ${ }^{16}$ San et al randomised treatment with haloperidol, olanzapine, risperidone, quetiapine and ziprasidone to 114 antipsychotic-naive FEP patients. The lowest discontinuation rate observed by 1 year was for olanzapine (40\%), this being significantly lower than for risperidone $(64 \%)$ and quetiapine (56.5\%) but all lower than haloperidol and ziprasidone (both $>80 \%) .{ }^{15}$ In a further naturalistic first-episode schizophrenia spectrum disorder study, Liew et al found significantly lower rates of discontinuation of risperidone compared with haloperidol and trifluoperazine in an Asian cohort (only risperidone was used commonly in our sample). ${ }^{17}$ The median time to discontinuation for risperidone was 69 days, shorter than we report.

\section{Limitations and strengths}

This study is one of the largest investigations reported of a realworld cohort of FEP patients taking a range of commonly used medications. It arguably reflects standard patients seen by early psychosis services across the UK, including seven separate teams with different geographical catchments (both urban and rural) and notable ethnic variation. It is possible that variation from findings of some previous studies ${ }^{15,16}$ reflects different patient populations. Although it is possible that our results incorporate bias of medication choice, due to the non-randomised nature of the study, this is mitigated by the number of services and prescribing doctors involved, the fact that none of the prescribers was aware that discontinuation time would be used as a method to compare medications and the non-significant effect of medication type on the all-cause discontinuation. The interrater reliability as to date of discontinuation was very high which suggests minimal rater bias.

A disadvantage of retrospective studies where case notes are relied upon is the potential for information bias. Clinical records have not been kept in a manner that was designed specifically for this study, and the data we required were not always measured and recorded in ways that were ideal. That said, there is only a small amount of missing follow-up data for those prescribed an antipsychotic (7\%) but we cannot be sure that the people whose data we could not locate did not have different discontinuation 


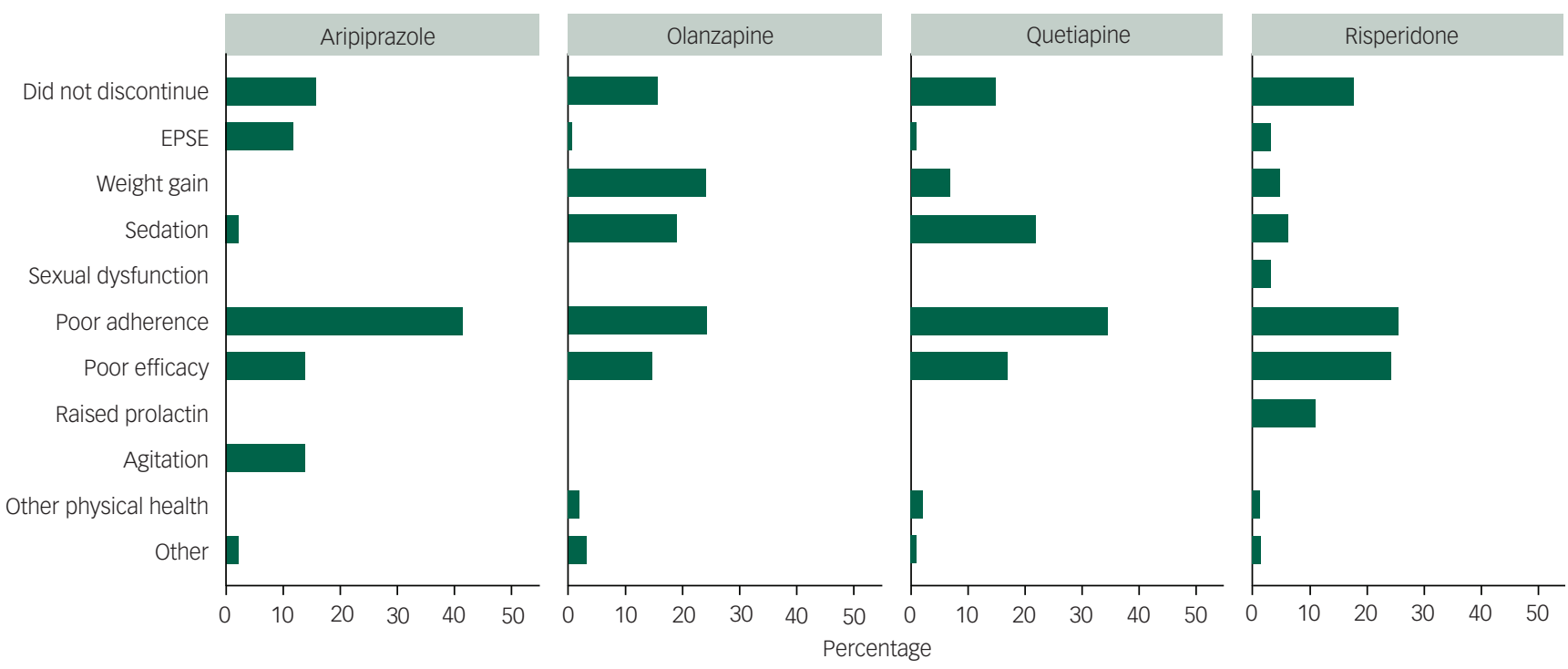

Fig. 3 Primary reason for treatment discontinuation by medication.

rates. The age, gender and DUP of those with missing data were not significantly different from the included cohort with full data available. Although our raters demonstrated good interrater reliability, the ratings on discontinuation are taken from the clinical files and therefore rely on the accuracy of these files. We were also not able to collect some information on possible confounders such as ethnicity, specific diagnosis (not just FEP), functional impairment and illness severity. We did not include a variable related to medication dose owing to the differences between medications and variation over time. All of the early intervention teams used NICE guideline interventions and lowdose medication strategies; three of the teams had a specific lowdose FEP service protocol. This may bias particularly against quetiapine due to its wide dosing flexibility and low dopamine D2 receptor affinity at lower doses. Further research in larger samples to investigate treatment groups with smaller numbers (such as aripiprazole in this study), and enable further subgroup analysis, is warranted. The documented cause of discontinuation is arguably the least reliable variable considered, with potential overlap of variables and bias by preconceived clinician and patient attitudes about the properties of a medication. This would require prospective qualitative analysis to explore more clearly, particularly in the 'poor adherence' coded group. Significant adverse effects differentially associated with the medications studied, and ranked by reported frequency, clearly reflect those from existing literature however. ${ }^{3,21}$ Recruitment to earlier studies also may influence patient and clinician attitude to medication with greater recent publicity around metabolic issues with olanzapine $v$. other medications.

We excluded individuals prescribed long-acting injectable (depot) medication but the numbers of patients started on this as a first treatment were small $(n=4)$ as per usual current treatment for FEP. The cohort has a low rate of individuals starting on other antipsychotics such as amisulpride or older dopamine antagonists and does not include other newer medications that have recently been licenced in the UK (asenapine and lurasidone).

\section{Clinical implications}

This naturalistic study by multivariable analysis, adjusting for confounding factors, assists in confirming the applicability of findings from existing randomised studies to the wider clinical setting. These studies build on efficacy studies to largely support a lack of difference in effectiveness (as measured by all-cause time to discontinuation) between commonly used antipsychotic medications in FEP. Most patients will discontinue their first antipsychotic medication (for a variety of reasons) in the first year of treatment however, and clinicians should expect this to be the norm rather than the exception. Strategies to improve this should be more widely adopted, such as shared decision-making and insight-orientated interventions. ${ }^{25}$ This is also clearly relevant beyond first episode prescribing. ${ }^{26}$ Long-acting antipsychotic preparations, not explored in this study, may also have a role in enhancing effectiveness in this setting. ${ }^{27}$ Common adverse effects associated with discontinuing specific medications, as highlighted by this study and others, ${ }^{21}$ should be a focus of discussion pre-treatment when deciding with the patient which medication should be first prescribed.

Richard Whale, MD, MRCPsych, Sussex Partnership NHS Foundation Trust, Brighton, UK, and Brighton and Sussex Medical School, Brighton, UK; Michael Harris, MSC, MRCPsych, Sussex Partnership NHS Foundation Trust, Brighton, UK; Gail Kavanagh, MRCPsych, Sussex Partnership NHS Foundation Trust, Brighton, UK; Vijitha Wickramasinghe, MSC, MBBS, Sussex Partnership NHS Foundation Trust, Eastbourne, UK; Christopher I. Jones, PhD, Brighton and Sussex Medical School, Brighton,

UK; Steven Marwaha, PhD, MRCPsych, Division of Mental Health and Wellbeing, University of Warwick, Coventry, UK, and Coventry and Warwickshire Partnership NHS Trust, Coventry, UK; Ketan Jethwa, PGCert, BMBS, Division of Mental Health and Wellbeing University of Warwick, Coventry, UK, and Coventry and Warwickshire Partnership NHS Trust, Coventry, UK; Nirmalan Ayadurai, MRCPsych, Coventry and Warwickshire Partnership NHS Trust, Coventry, UK; Andrew Thompson, MD, FRCPsych, Division of Mental Health and Wellbeing, University of Warwick, Coventry, UK, and Coventry and Warwickshire Partnership NHS Trust, Coventry, UK

Correspondence: Dr Richard Whale, Division of Medical Education, Brighton and Sussex Medical School, Mayfield House, Falmer, Brighton BN1 9PH, UK. Email: richard.whale@brighton.ac.uk

First received 28 Jan 2016, final revision 6 Sep 2016, accepted 12 Sep 2016

\section{References}

1 Leucht S, Pitschel-Walz G, Abraham D, Kissling W. Efficacy and extrapyramidal side-effects of the new antipsychotics olanzapine, quetiapine, risperidone, and sertindole compared to conventional antipsychotics and placebo. A meta-analysis of randomized controlled trials. Schizophr Res 1999; 35: 51-68.

2 Leucht S, Tardy M, Komossa K, Heres S, Kissling W, Salanti G, Davis JM. Antipsychotic drugs versus placebo for relapse prevention in schizophrenia: a systematic review and meta-analysis. Lancet 2012; 379: 2063-71. 
3 Leucht S, Cipriani A, Spineli L, Mavridis D, Orey D, Richter F, et al. Comparative efficacy and tolerability of 15 antipsychotic drugs in schizophrenia: a multipletreatments meta-analysis. Lancet 2013; 382: 951-62.

4 Crossley NA, Constante M, McGuire P, Power P. Efficacy of atypical v. typical antipsychotics in the treatment of early psychosis: meta-analysis. $\mathrm{Br} J$ Psychiatry 2010; 196: 434-9.

5 Fraguas D, Correll CU, Merchan-Naranjo J, Rapado-Castro M, Parellada M, Moreno $\mathrm{C}$, et al. Efficacy and safety of second-generation antipsychotics in children and adolescents with psychotic and bipolar spectrum disorders: comprehensive review of prospective head-to-head and placebo-controlled comparisons. Eur Neuropsychopharmacol 2011; 21: 621-45.

6 Zhang JP, Gallego JA, Robinson DG, Malhotra AK, Kane JM, Correll CU. Efficacy and safety of individual second-generation vs first-generation antipsychotics in firs episode psychosis: a systematic review and meta-analysis. Int I Neuropsychopharmacol 2013; 16: 1205-18.

7 Alvarez-Jimenez M, Gonzalez-Blanch C, Crespo-Facorro B, Hetrick S, Rodríguez Sánchez JM, Pérez-Iglesias R, et al. Antipsychotic-induced weight gain in chronic and first-episode psychotic disorders: a systematic critical reappraisal. CNS Drugs 2008; 22: 547-62.

8 Streiner DL. Statistics commentary series: commentary \#6 - are trial participants representative of 'real' patients. J Clin Psychopharm 2015; 35: 4-6.

9 Lieberman J, Stroup T, McEvoy J, Swartz MS, Rosenheck RA, Perkins DO, et al. Effectiveness of antipsychotic drugs in patients with chronic schizophrenia. N Eng/ Med 2005; 353: 1209-23

10 Kahn RS, Fleischhacker WW, Boter $\mathrm{H}$, Davidson M, Vergouwe $\mathrm{Y}$, Keet IP, et al. Effectiveness of antipsychotic drugs in first-episode schizophrenia and schizophreniform disorder: an open randomised clinical trial. Lancet 2008; 371: 1085-97.

11 Lewis SW, Barnes TR, Davies L, Murray RM, Dunn G, Hayhurst KP, et al. Randomized controlled trial of effect of prescription of clozapine versus other second-generation antipsychotic drugs in resistant schizophrenia. Schizophr Bull 2006; 32: 715-23

12 McEvoy JP, Lieberman JA, Perkins DO, Hamer RM, Gu H, Lazarus A, et al. Efficacy and tolerability of olanzapine, quetiapine, and risperidone in the treatment of early psychosis: a randomized, double-blind 52-week comparison. Am J Psychiatry 2007; 164: 1050-60.

13 Johnsen E, Kroken RA, Wentzel-Larsen T, Jørgensen HA. Effectiveness of secondgeneration antipsychotics: a naturalistic, randomized comparison of olanzapine, quetiapine, risperidone, and ziprasidone. BMC Psychiatry 2010; 10: 26.

14 Crespo-Facorro B, Perez-Iglesias R, Mata I, Ramirez-Bonilla M, Martínez-Garcia O, Pardo-Garcia G, et al. Effectiveness of haloperidol, risperidone and olanzapine in the treatment of first-episode non-affective psychosis: results of a randomized, flexible-dose, open-label 1-year follow-up comparison. J Psychopharmacol 2011; 25: 744-54.

15 San L, Arranz B, Perez V, Safont G, Corripio I, Ramirez N, et al. One-year, randomized, open trial comparing olanzapine, quetiapine, risperidone and ziprasidone effectiveness in antipsychInaive patients with a first-episode psychosis. Psychiatry Res 2012; 200: 693-701.

16 Tiihonen J, Haukka J, Taylor M, Haddad PM, Patel MX, Korhonen P. A nationwide cohort study of oral and depot antipsychotics after first hospitalization for schizophrenia. Am J Psychiatry 2011; 168: 603-9.

17 Liew A, Verma S, Lye Yin P, Edimansyah A, Subramaniam M, Vaingankar J, et al. Comparing effectiveness of risperidone with first-generation antipsychotic medications in patients with schizophrenia-spectrum disorders. J Psychopharmacol 2010; 24: 973-80

18 Olfson M, Gerhard T, Huang C, Lieberman JA, Bobo WV, Crystal S. Comparative effectiveness of second-generation antipsychotic medications in early-onset schizophrenia. Schizophr Bull 2012; 38: 845-53.

19 Lieberman JA, Stroup TS, McEvoy JP, Swartz MS, Rosenheck RA, Perkins DO. Clinical Antipsychotic Trials of Intervention Effectiveness (CATIE) Investigators. Effectiveness of antipsychotic drugs in patients with chronic schizophrenia. N Engl J Med 2005; 353: 1209-23.

20 Early Psychosis Guidelines Writing Group and EPPIC National Support Program. Australian Clinical Guidelines for Early Psychosis (2nd edn, update). Orygen, The National Centre of Excellence in Youth Mental Health, 2016.

21 Young SL, Taylor M, Lawrie SM. 'First do no harm'. A systematic review of the prevalence and management of antipsychotic adverse effects. J Psychopharmacol 2015; 29: 353-62.

22 Hill $M$, Crumlish N, Clarke M, Whitty P, Owens E, Renwick L, et al. Prospective relationship of duration of untreated psychosis to psychopathology and functional outcome over 12 years. Schizophr Res 2012; 141: 215-21.

23 Levine SZ, Leucht S. Delayed- and early-onset hypotheses of antipsychotic drug action in the negative symptoms of schizophrenia. Eur Neuropsychopharmacol 2012; 22: 812-7.

24 Crespo-Facorro B, Pérez-Iglesias R, Mata I, Martínez-Garcia O, Ortiz V PelayoTerán JM, et al. Long-term (3-year) effectiveness of haloperidol, risperidone and olanzapine: results of a randomized, flexible-dose, open-label comparison in firstepisode nonaffective psychosis. Psychopharmacology 2012; 219: 225-33.

25 Simmons MB, Hetrick SE. 'Prodromal' research and clinical services: The imperative for shared decision-making. Aust N Z J Psychiatry 2012; 46: 66.

26 Whale R, Pereira M, Cuthbert S, Fialho R. Effectiveness and predictors of continuation of paliperidone palmitate long acting injection treatment: a 12 month naturalistic cohort study. J Clin Psychopharm 2015; 35: 591-5.

27 Subotnik KL, Casaus LR, Ventura J, Luo JS, Hellemann GS, Gretchen-Doorly D, et al. Long-acting injectable risperidone for relapse prevention and control of breakthrough symptoms after a recent first episode of schizophrenia. A randomized clinical trial. JAMA Psychiatry 2015; 72: 822-9. 